\title{
Population pharmacokinetic model development and its relationship with adverse events of oxcarbazepine in adult patients with epilepsy
}

\section{Yoonhyuk Jang}

Seoul National University Hospital

\section{Seonghae Yoon}

Seoul National University Hospital

Tae-Joon Kim

Ajou University

SeungHwan Lee

Seoul National University Hospital

Kyung-Sang Yu

Seoul National University Hospital

In-Jin Jang

Seoul National University Hospital

Kon Chu ( $\nabla$ stemcell.snu@gmail.com )

Seoul National University Hospital

\section{Sang Kun Lee}

Seoul National University Hospital

\section{Research Article}

Keywords: oxcarbazepine, pharmacokinetic, adverse events, dose-related adverse events, epilepsy, antiepileptic drugs

Posted Date: January 13th, 2021

DOl: https://doi.org/10.21203/rs.3.rs-138619/v1

License: (c) (i) This work is licensed under a Creative Commons Attribution 4.0 International License. Read Full License

Version of Record: A version of this preprint was published at Scientific Reports on March 18th, 2021. See the published version at https://doi.org/10.1038/s41598-021-85920-0. 


\section{Abstract}

This study aimed to develop a pharmacokinetic (PK) model of oxcarbazepine (OXC) and analyse the relationship between monohydroxylated derivative (MHD), an active metabolite of OXC, and the adverse events of OXC. We obtained 711 OXC samples from 618 patients with epilepsy who were enrolled in the Epilepsy Registry Cohort of Seoul National University Hospital from February 2011 to January 2014. The plasma PK model was developed using a nonlinear mixed-effect modelling method with NONMEM (ver 7.3). A one-compartment model with a first-order absorption model and proportional residual error adequately described the MHD concentration-time profiles. The only covariate incorporated for $\mathrm{CL} / \mathrm{F}$ and V/F was body weight. Of the 447 patients analysed, 28 (6.26\%) had dose-related adverse events (DRAEs), which were dizziness, somnolence, headache, and diplopia. For DRAE occurrence, the cut-off values of the MHD trough and AUC were $12.27 \mathrm{mg} / \mathrm{L}$ (specificity 0.570 , sensitivity 0.643 ) and $698.5 \mathrm{mg} \cdot \mathrm{h} / \mathrm{L}$ (specificity, sensitivity 0.571 ), respectively. Multivariate analysis showed the sole dizziness symptom was significantly associated with both the MHD trough and the AUC ( $p=0.013, p=0.038$, respectively). We newly developed a population PK model using sparse sampling data from patients with epilepsy, and the model better reflects the actual clinical situation.

\section{Bullet Points}

- We developed a population PK model using sparse sampling data from patients with epilepsy, which better reflects the actual clinical situation.

- Of the 447 patients analysed, $28(6.26 \%)$ had dose-related adverse events, which were dizziness, somnolence, headache, and diplopia.

- For dose-related adverse events occurrence, the cut-off values of the MHD trough and AUC were $12.27 \mathrm{mg} / \mathrm{L}$ and $698.5 \mathrm{mg} \cdot \mathrm{h} / \mathrm{L}$, respectively.

- Multivariate analysis showed the sole dizziness symptom was significantly associated with both the MHD trough and the AUC.

\section{Introduction}

Oxcarbazepine (OXC) is an antiepileptic drug (AED) that is prevalently prescribed for patients with epilepsy. OXC, as a structural variation form of carbamazepine (CBZ), is an effective option for focal seizures as a monotherapy or adjuvant therapy and has fewer adverse events than CBZ. ${ }^{1-3}$

OXC is rapidly absorbed and almost completely converted to its active metabolite 10,11-dihydro-10hydroxy-carbamazepine (monohydroxy derivative, MHD), which has a major role in the antiepileptic effect in humans. Whereas only $2 \%$ of total radioactivity in plasma is due to unchanged $\mathrm{OXC}$, more than $70 \%$ is attributed to MHD metabolized by cytosolic aryl ketone reductase in the liver. ${ }^{4-6}$ Thus, for patients who take OXC, exposure to MHD is approximately 15 times higher than exposure to OXC. ${ }^{7}$ OXC and MHD exert their antiepileptic activity by blocking voltage-sensitive sodium channels and stabilizing hyperexcited 
neuronal membranes. ${ }^{8}$ OXC is cleared mostly in the form of metabolites, which are excreted by the kidneys. In addition, OXC is known to have drug-drug interactions, acting as a perpetrator and substrate at the same time. Several studies suggest that cytochrome P450 (CYP) isoenzyme-inducing drugs (e.g., phenytoin, phenobarbital, carbamazepine, EIAEDs) decrease the concentration of MHD, which may require an increase in the $\mathrm{OXC}$ dose to maintain drug effects. $6,9,10$

Serum MHD levels also play a critical role in the occurrence of adverse events induced by OXC. The adverse events of OXC include dizziness, diplopia, ataxia, skin rash, and hyponatremia, ${ }^{3,11}$ and the different pathomechanisms are known to be associated with adverse events. Maculopapular eruption, which is one of the most serious events of $O X C$, is associated with autoimmune reactions involving specific subtypes of human leukocyte antigen. ${ }^{12}$ In contrast, dizziness, the most common adverse event of OXC, is dependent on MHD serum levels, ${ }^{13}$ implying that pharmacokinetics (PK) of OXC may play an important role. Additionally, dose-related adverse events (DRAEs) are known to include dizziness, somnolence, headache, and diplopia; ${ }^{3}$ however, the specific cut-off levels or PK variables of OXC and MHD that cause DRAEs have not been studied previously.

In this study, we developed a population PK model of MHD to explore various covariates, including comedications. We also investigated adverse events related to OXC and the relationship between PK and these adverse events.

\section{Methods}

\section{Patient enrolment, samples and assays}

We obtained 711 OXC samples from 618 patients with epilepsy who were enrolled in the Epilepsy Registry Cohort of Seoul National University Hospital from February 2011 to January 2014. We collected peripheral blood samples at a steady state level of the drug in the circulation. Serial blood samples were obtained from the single-dose study at $2,4,6,8,12,14,16$, and 24 hours post dose. The actual dosing and sampling time were used in the analysis. Plasma concentrations of MHD were determined using the validated liquid chromatography-tandem mass spectrometry method. ${ }^{7}$

Variables including body weight, final dosing time, and blood sampling time were obtained and available in 447 patients whose data were used for further analysis. To develop a PK model, the data of the PK study involving 40 patients evaluating a $30 \mathrm{mg} / \mathrm{kg}$ single oral dose of OXC were combined. ${ }^{7}$ The retrospective review was performed from each patient's medical records, including OXC dosing histories, times of blood sampling, seizure frequency, reported adverse events, coadministered AEDs and their regimen, and routine laboratory results. Seizure-free was defined as having no epileptic seizure for at least 3 months before the blood sample was taken. The existence of dizziness, somnolence, diplopia, tremor, ataxia, dysarthria, encephalopathy, cerebral atrophy, psychiatric symptoms, fatigue, headache, visual field defect, epistaxis, skin rash, haematologic effects, liver function test abnormalities, hyponatremia, reproductive problems, cardiovascular complications, gastrointestinal problems, body 
weight changes, paresthesia, renal stone, osteoporosis, acne, hirsutism, hair loss, gingival hyperplasia, and limb oedema was checked as adverse events of OXC administration. Among them, dizziness, somnolence, headache, and diplopia were defined as DRAEs. ${ }^{3}$

This study was approved by the Institutional Review Board of Seoul National University (IRB No. 1010042-335, 12/06/2010). Written informed consent was obtained from all patients. All methods were performed in accordance with the relevant guidelines and regulations. Informed consent was obtained for all the subjects who are under 18 , from a parent and/or legal guardian.

\section{Population PK model development and model evaluation}

We developed a population PK model of MHD using a nonlinear mixed-effects modelling method with NONMEM, version 7.3. A total of 748 data points of 487 patients were used in this analysis. We assumed that MHD followed a one-compartment model with first-order elimination, which was also adopted in other studies. The ADVAN2 subroutine and first-order conditional estimation with the interaction method were used. The absorption rate $(\mathrm{ka})$, apparent clearance $(\mathrm{CL} / \mathrm{F})$ and apparent volume of distribution (V/F) were estimated. The interindividual variability (IIV) associated with PK parameters was modelled using an exponential model as follows:

\section{$\theta_{i}=\theta_{\text {typical }} \times e^{\eta_{i}}$}

where $\theta_{i}$ is the parameter for the $\mathrm{i}^{\text {th }}$ subject, $\theta_{\text {typical }}$ is the typical value of the parameter, and $\eta_{i}$ is a normally distributed random variable for the $\mathrm{i}^{\text {th }}$ subject, with a mean of zero and variance $\omega^{2}$. Several error models, including additive, proportional, and combined error models, were tested, and a proportional error model was used in the final model.

The effects of covariates such as age, sex, and concomitant drugs were explored. Body weight was incorporated as a covariate for $\mathrm{CL} / \mathrm{F}$ and $\mathrm{Vd}$ considering its relationship with PK parameters. ${ }^{14-16}$ The effects of other antiepileptic drugs (phenytoin, valproate, phenobarbital, lamotrigine, pregabalin, topiramate, zonisamide, levetiracetam, clobazan, vigabatrin, lorazepam) or comedication with enzymeinducing antiepileptic drugs (carbamazepine, phenytoin, or phenobarbital) were evaluated. Stepwise forward selection and a backward elimination method were used. The objective function value (OFV) decrease of 3.84 ( $\chi^{2}$ distribution, $p<0.05$ ) was considered significant in the forward selection process; the OFV increase of $6.63(p<0.01)$ was considered significant in the backward elimination process.

The adequacy of each model was evaluated based on not only OFV but also goodness-of-fit plots. Plots of observed vs predicted values of individual and population values were evaluated for randomness around the line of unity. Plots of conditional weighted residual (CWRES) vs. population predicted values and time after dose were evaluated for randomness around the zero line. To evaluate the stability and robustness of the final PK model, a bootstrap resampling method was used. Resampling with replacement generated 1,000 bootstrap datasets, and the final population PK model was fitted to each of 
these datasets. The median and $95 \%$ confidence intervals of parameters obtained from this step were compared with the final parameter estimates. A visual prediction check was also performed using a graphical comparison of the simulated data overlaid with the observed data.

\section{Statistics}

Clinical data are presented as the mean \pm standard deviation or number with percentage. For group comparisons, we utilized Mann-Whitney U tests or Fisher's exact tests for continuous or categorical variables. The difference in PK variables between the groups with and without adverse events was analysed with the Wilcoxon matched pairs signed-rank test. R version 4.0.2 (R Foundation for Statistical Computing, Vienna, Austria) was used for analysis, and a $p$-value $<.05$ was considered statistically significant. The optimal cut-off value of the MHD levels for the prediction of adverse events was identified using the receiver operating characteristic (ROC) curve (R package pROC).

\section{Results}

\section{Patient characteristics}

Of the 447 patients analysed, two hundred sixty-four (59.1\%) were male (Table 1). The mean age was 39.2 years (range 16 to 80 ), and the average body weight was 65.8 kilograms (range 39 to 116). The number of patients who were administered the combination therapy was $292(65.3 \%)$, and on average, they took two AEDs, including OXC (interquartile 1 to 3 ). The most common coadministered AED was levetiracetam $(162,55.5 \%)$, followed by topiramate $(96,32.9 \%)$. Among the patients taking multiple AEDs, only $23(5.1 \%)$ had EIAEDs. In total, $63.8 \%$ (285) of the patients were seizure-free, and the daily OXC dose was $999 \mathrm{mg}$ on average (range 150 to 3300 ). 
Table 1

Characteristics of patients taking oxcarbazepine according to the occurrence of dose-related adverse events

\begin{tabular}{|c|c|c|c|c|c|c|c|}
\hline \multirow[t]{2}{*}{ Parameter } & \multirow{2}{*}{$\begin{array}{l}\text { Total }(\mathrm{N} \\
=447)\end{array}$} & \multicolumn{3}{|c|}{ Dose-related adverse events } & \multicolumn{3}{|c|}{ Only dizziness } \\
\hline & & $\begin{array}{l}A E+(n= \\
28)\end{array}$ & $\begin{array}{l}A E-(n= \\
419)\end{array}$ & $\begin{array}{l}P \\
\text { value }\end{array}$ & $\begin{array}{l}\mathrm{Dz}+(\mathrm{n} \\
=18)\end{array}$ & $\begin{array}{l}\text { Dz- }(n= \\
429)\end{array}$ & $\begin{array}{l}P \\
\text { value }\end{array}$ \\
\hline \multicolumn{8}{|l|}{ Demographic } \\
\hline Age (yrs) & $\begin{array}{l}39.2 \pm \\
14.7\end{array}$ & $\begin{array}{l}37.3 \pm \\
14.8\end{array}$ & $\begin{array}{l}39.3 \pm \\
14.7\end{array}$ & 0.449 & $\begin{array}{l}34.9 \pm \\
13.1\end{array}$ & $\begin{array}{l}39.4 \pm \\
14.7\end{array}$ & 0.211 \\
\hline Sex, male & $\begin{array}{l}264 \\
(59.1 \%)\end{array}$ & $\begin{array}{l}14 \\
(50.0 \%)\end{array}$ & $\begin{array}{l}250 \\
(59.7 \%)\end{array}$ & 0.327 & $\begin{array}{l}10 \\
(55.6 \%)\end{array}$ & $\begin{array}{l}254 \\
(59.2 \%)\end{array}$ & 0.809 \\
\hline $\begin{array}{l}\text { Body weights } \\
(\mathrm{kg})\end{array}$ & $\begin{array}{l}65.8 \pm \\
12.5\end{array}$ & $\begin{array}{l}63.7 \pm \\
12.2\end{array}$ & $\begin{array}{l}65.9 \pm \\
12.5\end{array}$ & 0.489 & $\begin{array}{l}68.2 \pm \\
9.6\end{array}$ & $\begin{array}{l}65.7 \pm \\
12.6\end{array}$ & 0.235 \\
\hline \multicolumn{8}{|l|}{$\begin{array}{l}\text { Clinical } \\
\text { information }\end{array}$} \\
\hline No. of $A E D s^{\dagger}$ & $2[1-3]$ & $2[1-3]$ & $2[1-3]$ & 0.375 & $2[1-3]$ & $2[1-3]$ & 0.950 \\
\hline Co-AEDs & $\begin{array}{l}292 \\
(65.3 \%)\end{array}$ & $\begin{array}{l}19 \\
(67.9 \%)\end{array}$ & $\begin{array}{l}273 \\
(65.2 \%)\end{array}$ & 0.840 & $\begin{array}{l}11 \\
(61.1 \%)\end{array}$ & $\begin{array}{l}281 \\
(65.5 \%)\end{array}$ & 0.801 \\
\hline Co-EIAEDs & $23(5.1 \%)$ & $2(7.1 \%)$ & $\begin{array}{l}21 \\
(5.0 \%)\end{array}$ & 0.648 & 0 & $\begin{array}{l}23 \\
(5.4 \%)\end{array}$ & 0.614 \\
\hline Seizure-free & $\begin{array}{l}285 \\
(63.8 \%)\end{array}$ & $\begin{array}{l}13 \\
(46.4 \%)\end{array}$ & $\begin{array}{l}272 \\
(64.9 \%)\end{array}$ & 0.066 & 8 & $\begin{array}{l}277 \\
(64.6 \%)\end{array}$ & 0.130 \\
\hline \multicolumn{8}{|l|}{ OXC PK } \\
\hline OXC dose (mg) & $\begin{array}{l}999 \pm \\
338\end{array}$ & $\begin{array}{l}1130 \pm \\
512\end{array}$ & $\begin{array}{l}990 \pm \\
322\end{array}$ & 0.181 & $\begin{array}{l}1216 \pm \\
598\end{array}$ & $\begin{array}{l}989 \pm \\
321\end{array}$ & 0.053 \\
\hline $\begin{array}{l}\text { MHD trough } \\
\text { level (mg/L) }\end{array}$ & $\begin{array}{l}12.75 \pm \\
7.17\end{array}$ & $\begin{array}{l}15.24 \pm \\
8.26\end{array}$ & $\begin{array}{l}12.58 \pm \\
7.08\end{array}$ & 0.086 & $\begin{array}{l}17.48 \pm \\
8.89\end{array}$ & $\begin{array}{l}12.55 \pm \\
7.04\end{array}$ & $0.013^{\star}$ \\
\hline $\begin{array}{l}\text { MHD AUC } \\
(\mathrm{mg} \cdot \mathrm{h} / \mathrm{L})\end{array}$ & $\begin{array}{l}653.8 \pm \\
268.7\end{array}$ & $\begin{array}{l}753.1 \pm \\
285.3\end{array}$ & $\begin{array}{l}647.2 \pm \\
266.6\end{array}$ & $0.045^{*}$ & $\begin{array}{l}806.1 \pm \\
314.0\end{array}$ & $\begin{array}{l}647.5 \pm \\
265.1\end{array}$ & $0.022^{*}$ \\
\hline
\end{tabular}




\begin{tabular}{|c|c|c|c|c|c|c|c|}
\hline \multirow[t]{2}{*}{ Parameter } & \multirow{2}{*}{$\begin{array}{l}\text { Total }(\mathrm{N} \\
=447)\end{array}$} & \multicolumn{3}{|c|}{ Dose-related adverse events } & \multicolumn{3}{|c|}{ Only dizziness } \\
\hline & & $\begin{array}{l}A E+(n= \\
28)\end{array}$ & $\begin{array}{l}\mathrm{AE}-(\mathrm{n}= \\
419)\end{array}$ & $\begin{array}{l}P \\
\text { value }\end{array}$ & $\begin{array}{l}\mathrm{Dz}+(\mathrm{n} \\
=18)\end{array}$ & $\begin{array}{l}D z-(n= \\
429)\end{array}$ & $\begin{array}{l}P \\
\text { value }\end{array}$ \\
\hline \multicolumn{8}{|c|}{ Values are mean \pm SD or number (\%). } \\
\hline \multicolumn{8}{|l|}{${ }^{*} \mathrm{P}<0.050$} \\
\hline \multicolumn{8}{|c|}{${ }^{\dagger}$ Values in median [interquartile range] } \\
\hline \multicolumn{8}{|c|}{$\begin{array}{l}\text { Abbreviations: AE+, patients with dose-related adverse events; AE-, patients without dose-related } \\
\text { adverse events; Dz+, patients with only dizziness; Dz-, patients without only dizziness; AEDs, } \\
\text { antiepileptic drugs; Co-AEDs, existence of concomitant AEDs; Co-EIAEDs, existence of concomitant } \\
\text { enzyme-inducing AEDs ( } \leq 6) \text {; OXC, oxcarbazepine; PK, pharmacokinetics; MHD, Monohydroxy } \\
\text { derivative of oxcarbazepine; AUC, area under the plasma concentration-time curve. }\end{array}$} \\
\hline \multicolumn{8}{|c|}{ Statistical analysis: Fisher exact test or Mann-Whitney's U test } \\
\hline
\end{tabular}

\section{Population PK analysis}

A one-compartment model with a first-order absorption model and proportional residual error adequately described the MHD concentration-time profiles. We explored several covariates, including age, body weight, sex, and concomitant drugs. The only covariate incorporated for $\mathrm{CL} / \mathrm{F}$ and V/F was body weight. The use of EIAEDs (carbamazepine, phenytoin, phenobarbital, or valproic acid) was tested as a covariate, and $\mathrm{CL} / \mathrm{F}$ increased $7 \%$ in the patients using EIAEDs. However, incorporation of the use of EIAEDs as a covariate did not improve the model based on OFV and the goodness-of-fit plot; thus, the use of EIAEDs was not included as a covariate in the final model. The final parameter estimates for $C L / F, V / F$, and ka were $1.65 \mathrm{~L} / \mathrm{h}, 59.0 \mathrm{~L}$, and $0.34 \mathrm{~h}^{-1}$, respectively (Table 2). The basic goodness-of-fit plots (Fig. 1) showed no pronounced bias, which means that the final model adequately described the data. 
Table 2

Parameter estimates and variability for the population pharmacokinetic model of MHD

\section{Parameters}

Estimates

(RSE)
Bootstrap median

$(95 \% \mathrm{Cl})$

Structural model

$\mathrm{CL} / \mathrm{F}=\theta 1 \times(\mathrm{WT} / 66)^{\theta 2} ;$ apparent clearance $\left(\mathrm{L} \cdot \mathrm{h}^{-1}\right)$

$\theta 1 ; C L / F$ typical value $\left(L \cdot h^{-1}\right)$

$1.65(1.8 \%)$

$1.65(1.59-1.70)$

$\theta 2$; body weight exponent

$0.67(14.2 \%)$

$0.66(0.47-0.85)$

$\mathrm{Vd} / \mathrm{F}(\mathrm{L})=\theta 3 \times(\mathrm{WT} / 66)^{\theta 4}$; apparent volume of distribution

$(\mathrm{L})$

$\theta 3 ; \mathrm{Vd} / \mathrm{F}$ typical value $\left(\mathrm{L} \cdot \mathrm{h}^{-1}\right)$

$59.0(4.5 \%)$

$59.2(54.2-64.8)$

$\theta 4$; body weight exponent

$0.96(18.1 \%)$

$0.95(0.59-1.3)$

ka; absorption rate constant $\left(\mathrm{h}^{-1}\right)$

$0.34(9.7 \%)$

$0.34(0.28-0.41)$

Inter-individual variability (IIV)

IIV CL/F (\%CV)

$29.2(5.4 \%)$

$29.7(26.2-32.9)$

IIV V2/F (\%CV)

$41.5(14.3 \%)$

$42.5(29.8-57.3)$

Residual error

Proportional error (SD; $\left.\mathrm{ng} \cdot \mathrm{mL}^{-1}\right)$

$0.13(10.8 \%)$

$0.13(0.10-0.16)$

${ }^{a} \mathrm{Cl}$ : confidence interval, which was estimated by applying the final population pharmacokinetic model to 1000 resampled datasets, CV: coefficient of variation, MHD: monohydroxy derivative of oxcarbazepine, RSE: relative standard error, SD: standard deviation, WT: body weight

The median parameter estimates obtained from the bootstrap analysis were similar to those of the final model, indicating acceptable precision of the final model (Table 2). In visual predictive checks, the median and $90 \%$ interpercentile range curves of the simulated concentrations adequately predicted the PK profiles of MHD (Supplementary Fig. 1).

\section{Adverse events of Oxcarbazepine}

A total of 33 patients (7.38\%) experienced at least one adverse event during OXC administration. Dizziness $(24,72.7 \%)$ was the most common symptom among the adverse events, followed by hyponatremia $(4,12.1 \%)$, which showed $132.5 \mathrm{mEq} / \mathrm{L}$ on average (range 126 to 142 ). Three patients $(9.09 \%)$ suffered from somnolence. Otherwise, skin rash, diplopia, and headache were shown in two patients (6.06\%). Tremor, dysarthria, and epistaxis were also reported in one patient (3.03\%). 


\section{Association of oxcarbazepine adverse events with MHD level}

To elucidate the relationship between OXC and MHD levels and OXC adverse events, we analysed the groups that had adverse events. Adverse events were defined into two groups: the group with DRAEs, including dizziness, somnolence, headache, or diplopia, and the group who reported only dizziness (Table 1). For the patients with DRAEs $(n=28)$, the OXC dose and MHD trough level showed a tendency to be higher than those of the patients without DRAEs, but the difference was not statistically significant ( 1130 vs $990, p=0.181,15.24$ vs $12.58, p=0.086$, respectively). However, the MHD area under the plasma concentration-time curve (AUC) was significantly higher in the DRAE group (753.1 vs 647.2, $p=0.045$ ). MHD trough and MHD AUC showed a significant association with dizziness adverse events (17.48 vs $12.55, p=0.013,806.1$ vs $647.5, p=0.022$, respectively). The OXC dose was also higher in the patients who showed only dizziness, but it did not reach statistical significance (1216 vs 989, $p=0.053$ ).

Using ROC curves, we estimated the cut-off value of MHD for the occurrence of adverse events. For DRAEs, the cut-off values of MHD AUC and trough levels were $698.5 \mathrm{mg} \cdot \mathrm{h} / \mathrm{L}$ (specificity, sensitivity 0.571) and $12.27 \mathrm{mg} / \mathrm{L}$ (specificity 0.570 , sensitivity 0.643 ), respectively (Fig. 2). For dizziness occurrence, the cut-off values of MHD were higher than those for DRAE; the MHD AUC level was $940.5 \mathrm{mg} \cdot \mathrm{h} / \mathrm{L}$ (specificity 0.872 , sensitivity 0.500 ), and the trough level was $19.15 \mathrm{mg} / \mathrm{L}$ (specificity 0.855 , sensitivity 0.500 ).

Multivariate analysis was performed to validate the association of the MHD trough and MHD AUC with adverse events (Table 3). Since OXC and its derivative are mainly excreted by the kidney, the glomerular filtration rate was also considered in the analysis. In the DRAE group, neither the MHD trough nor the MHD AUC showed a significant association ( $p=0.056$ and $p=0.062$, respectively). However, the sole dizziness symptom showed a significant association with both MHD trough and MHD AUC $(p=0.013$, OR $1.079,95 \% \mathrm{Cl} 1.016-1.145, \mathrm{p}=0.038, \mathrm{OR} 1.002, \mathrm{Cl} 1.000-1.1003$, respectively). 
Table 3

Multivariable analysis adjusted for age, sex, and GFR

\begin{tabular}{|c|c|c|c|c|c|c|c|c|}
\hline & \multicolumn{4}{|c|}{$\begin{array}{l}\text { Dose-related adverse events }(n= \\
\text { 28) }\end{array}$} & \multicolumn{4}{|c|}{ Only dizziness $(n=18)$} \\
\hline & OR & $\begin{array}{l}\text { P- } \\
\text { value }\end{array}$ & OR & $\begin{array}{l}\text { P- } \\
\text { value }\end{array}$ & OR & $\begin{array}{l}\text { P- } \\
\text { value }\end{array}$ & OR & $\begin{array}{l}\text { P- } \\
\text { value }\end{array}$ \\
\hline Sex & 1.001 & 0.968 & 1.001 & 0.967 & 0.976 & 0.268 & 0.977 & 0.275 \\
\hline Age & 1.202 & 0.689 & 1.214 & 0.672 & 0.921 & 0.887 & 0.984 & 0.978 \\
\hline GFR & 1.002 & 0.877 & 1.001 & 0.929 & 0.989 & 0.378 & 0.988 & 0.359 \\
\hline $\begin{array}{l}\text { MHD } \\
\text { trough }\end{array}$ & 1.051 & 0.056 & & & $\begin{array}{l}1.079 \\
(1.016- \\
1.145)^{\dagger}\end{array}$ & $0.013^{*}$ & & \\
\hline $\begin{array}{l}\text { MHD } \\
\text { AUC }\end{array}$ & & & 1.001 & 0.062 & & & $\begin{array}{l}1.002 \\
(1.000- \\
1.003)^{\dagger}\end{array}$ & $0.038 *$ \\
\hline \multicolumn{9}{|c|}{${ }^{*} \mathrm{P}<0.050$} \\
\hline \multicolumn{9}{|c|}{$\begin{array}{l}\text { Abbreviations: GFR, glomerular filtration rate; MHD, Monohydroxy derivative of oxcarbazepine; AUC, } \\
\text { area under the plasma concentration-time curve }\end{array}$} \\
\hline
\end{tabular}

\section{Discussion}

We developed a population PK model of MHD, the major metabolite of OXC in patients with epilepsy. In addition, the trough level and AUC were estimated from the final PK model to analyse the relationship between PK variables and adverse events of OXC.

As with previous population PK models, ${ }^{10,17-21}$ we developed a population PK model using MHD serum concentrations. Some studies included OXC itself as well as MHD in model development, ${ }^{19,20}$ but OXC is rapidly absorbed and almost completely converted into MHD, which is the major active metabolite in the human body. Moreover, exposure to OXC is negligibly small compared to MHD, so our study did not include OXC in the model development.

Body weight was incorporated as the only covariate for $\mathrm{CL} / \mathrm{F}$ and $\mathrm{V} / \mathrm{F}$ in our model. In most studies, the use of EIAEDs (carbamazepine, phenytoin, phenobarbital, and/or valproic acid) was a major covariate, showing increased CL/F from 17 to $30 \%$ in patients with EIAEDs. ${ }^{10,17,20,21}$ However, CL/F increased $7 \%$ in our study when incorporated as a covariate, and it did not significantly improve the model based on several diagnostic methods, such as OFV change and goodness-of-fit plots. According to in vitro and in vivo studies, OXC and MHD are competitive inhibitors of CYP2C19 and inducers of CYP3A4/5, which can lead to changes in plasma concentrations of other drugs, whereas the plasma concentrations of OXC and 
MHD can be affected by EIAEDs. ${ }^{6,9,10}$ However, the use of EIAEDs did not show a significant difference in exposure in our study, which means that EIAEDs may not actually have a decisive effect on the dose of $\mathrm{OXC}$ and MHD in the clinical setting.

While most studies used the population PK model to explore PK characteristics of OXC and MHD and analysed drug interactions with other AEDs, we investigated the relationship of the MHD level and the adverse events of OXC. The DRAEs showed a tendency to have higher MHD trough levels and AUCs but were not statistically significant. On the other hand, the sole dizziness symptom showed a significant correlation with both the MHD trough level and AUC. This result implies that the sole dizziness symptom could be associated with the MHD level in a dose-dependent manner. It is meaningful that our study quantitatively evaluated the relationship between drug serum levels and the DRAEs of OXC, which has never been rigorously validated before.

Dizziness is a complex term of various subjective symptoms, including "vertigo", "light-headedness", "unsteadiness", and "wooziness", reported by patients with drug adverse events. To differentiate the complaint into a homogenous feature, we focused on "wooziness", excluding vertigo, light-headedness, and unsteadiness, which were accompanied by diplopia, somnolence, and tremor. Patients with OXCinduced hyponatremia also experience dizziness, ${ }^{11}$ so patients who suffered from both dizziness and hyponatremia were excluded. In this respect, the sole dizziness reported by the patients in our study might reflect an aspect of the pure symptom "wooziness" induced by OXC overdose.

Our study has several limitations in that the number of patients was too small to evaluate the relationship between symptoms of DRAEs other than dizziness and MHD serum levels. Additionally, since this study was based on a retrospective medical review, adverse events might not be fully reported by the patients. During the prospective blood sampling of the patients, the AED dosage timing varied from patient to patient, and the interval between AED dosage time and blood sampling was not controlled. Nevertheless, the population PK model adequately described the PK of OXC in patients with epilepsy using sparse sampling, which is closely related to data from real practice.

In conclusion, we developed a new population PK model using sparse sampling data from patients with epilepsy. Our model better reflects the actual clinical situation than the previous model and is useful for choosing an appropriate dosage regimen for patients with epilepsy. In particular, the cut-off value of the MHD serum concentration by sparse samples would be helpful to assess the DRAEs of OXC in practice. We anticipate that further studies of the PK variable will also help in the DRAE monitoring of new AEDs.

\section{Declarations}

\section{Acknowledgements}

This work was supported by Samjin Pharmaceutical (0620201110).

\section{Ethical Publication Statement}


We confirm that we have read the journal's position on issues involved in ethical publication and affirm that this report is consistent with those guidelines.

\section{Data Availability Statement}

The data that support the findings of this study are available from the corresponding author upon reasonable request.

\section{Author Contributions}

Y.J., S.Y., and T-J.K. wrote and revised the manuscript. Y.J., S.Y., and T-J.K. analysed the data. Y.J., S.Y., and T-J.K. prepared the figures and tables. T-J.K., S.L., K-S.Y., and I-J.J. collected the samples and raw data. S.L., K-S.Y., I-J.J., K.C., and S.K.L. conceptualized and administered the study. K.C., and S.K.L. critically reviewed the manuscript.

\section{Conflicts of Interests}

The authors declare no conflict of interests.

\section{References}

1 Glauser, T. A. et al. Adjunctive therapy with oxcarbazepine in children with partial seizures. Neurology54, 2237, doi:10.1212/WNL.54.12.2237 (2000).

2 Schachter, S. C. et al. Oxcarbazepine: double-blind, randomized, placebo-control, monotherapy trial for partial seizures. Neurology52, 732-737, doi:10.1212/wnl.52.4.732 (1999).

3 Shorvon, S. Oxcarbazepine: a review. Seizure9, 75-79, doi:10.1053/seiz.2000.0391 (2000).

4 Schütz, H., Feldmann, K. F., Faigle, J. W., Kriemler, H. P. \& Winkler, T. The metabolism of 14Coxcarbazepine in man. Xenobiotica16, 769-778, doi:10.3109/00498258609043567 (1986).

5 Flesch, G. Overview of the clinical pharmacokinetics of oxcarbazepine. Clin Drug Investig24, 185-203, doi:10.2165/00044011-200424040-00001 (2004).

6 May, T. W., Korn-Merker, E. \& Rambeck, B. Clinical pharmacokinetics of oxcarbazepine. Clin Pharmacokinet42, 1023-1042, doi:10.2165/00003088-200342120-00002 (2003).

$7 \mathrm{Kim}$, D. W. et al. Efficacy, tolerability, and pharmacokinetics of oxcarbazepine oral loading in patients with epilepsy. Epilepsia53, e9-12, doi:10.1111/j.1528-1167.2011.03318.x (2012).

8 Trileptal (oxcarbazepine) [package insert]. East Hanover, NJ. Novartis Pharmaceutical Corp.;2000.

9 Glauser, T. A. Oxcarbazepine in the treatment of epilepsy. Pharmacotherapy21, 904-919, doi:10.1592/phco.21.11.904.34513 (2001). 
10 Wang, Y. et al. Population pharmacokinetics modeling of oxcarbazepine to characterize drug interactions in Chinese children with epilepsy. Acta Pharmacologica Sinica35, 1342-1350, doi:10.1038/aps.2014.76 (2014).

$11 \mathrm{Kim}, \mathrm{Y}$. S. et al. Frequency of and risk factors for oxcarbazepine-induced severe and symptomatic hyponatremia. Seizure23, 208-212, doi:10.1016/j.seizure.2013.11.015 (2014).

12 Moon, J. et al. HLA-B*40:02 and DRB1*04:03 are risk factors for oxcarbazepine-induced maculopapular eruption. Epilepsia57, 1879-1886, doi:10.1111/epi.13566 PMID - 27666425 (2016).

13 Sattler, A., Schaefer, M. \& May, T. W. Relationship between mono-hydroxy-carbazepine serum concentrations and adverse effects in patients on oxcarbazepine monotherapy. Seizure31, 149-154, doi:https://doi.org/10.1016/j.seizure.2015.07.018 (2015).

14 Anderson, B. J. \& Holford, N. H. Mechanism-based concepts of size and maturity in pharmacokinetics. Annu Rev Pharmacol Toxicol48, 303-332, doi:10.1146/annurev.pharmtox.48.113006.094708 (2008).

15 Anderson, B. J. \& Holford, N. H. G. Mechanistic Basis of Using Body Size and Maturation to Predict Clearance in Humans. Drug Metabolism and Pharmacokinetics24, 25-36, doi:https://doi.org/10.2133/dmpk.24.25 (2009).

16 McLeay, S. C., Morrish, G. A., Kirkpatrick, C. M. \& Green, B. The relationship between drug clearance and body size: systematic review and meta-analysis of the literature published from 2000 to 2007. Clin Pharmacokinet51, 319-330, doi:10.2165/11598930-000000000-00000 (2012).

17 Park, K. J. et al. Drug interaction and pharmacokinetic modeling of oxcarbazepine in korean patients with epilepsy. Clin Neuropharmaco/35, 40-44, doi:10.1097/WNF.0b013e31824150a5 (2012).

18 Peng, J., Zhang, H. N., Liu, Z. S., Xu, H. \& Wang, Y. Population pharmacokinetics of oxcarbazepine active metabolite in Chinese children with epilepsy. Int J Clin Pharmacol Ther52, 684-692, doi:10.5414/cp202078 (2014).

19 Antunes, N. J. et al. Population pharmacokinetics of oxcarbazepine and its metabolite 10hydroxycarbazepine in healthy subjects. Eur J Pharm Sci109s, S116-s123, doi:10.1016/j.ejps.2017.05.034 (2017).

20 Rodrigues, C. et al. Population pharmacokinetics of oxcarbazepine and its monohydroxy derivative in epileptic children. Br J Clin Pharmaco/83, 2695-2708, doi:10.1111/bcp.13392 (2017).

21 Faison, S. et al. Predicted Efficacy of Once-Daily Extended-Release Oxcarbazepine (Oxtellar XR(®)) Monotherapy in Adults and Children with Partial-Onset Seizures: Exposure-Response Modeling and Simulation. Clin Pharmaco/12, 135-147, doi:10.2147/CPAA.S256972 (2020). 


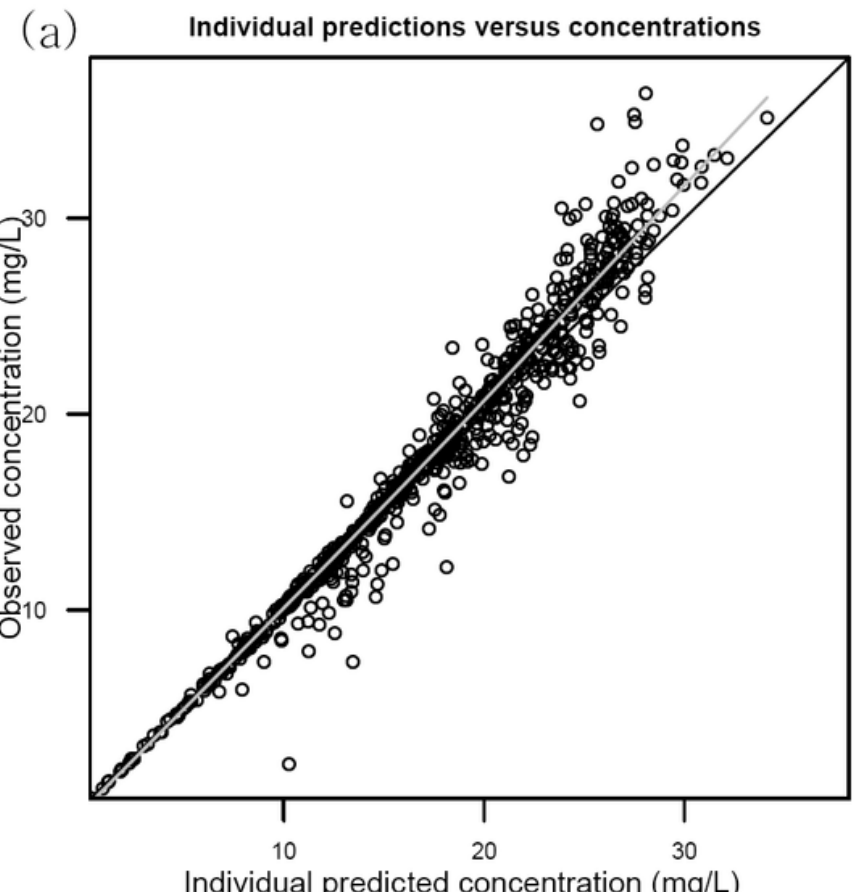

Individual predicted concentration ( $\mathrm{mg} / \mathrm{L})$

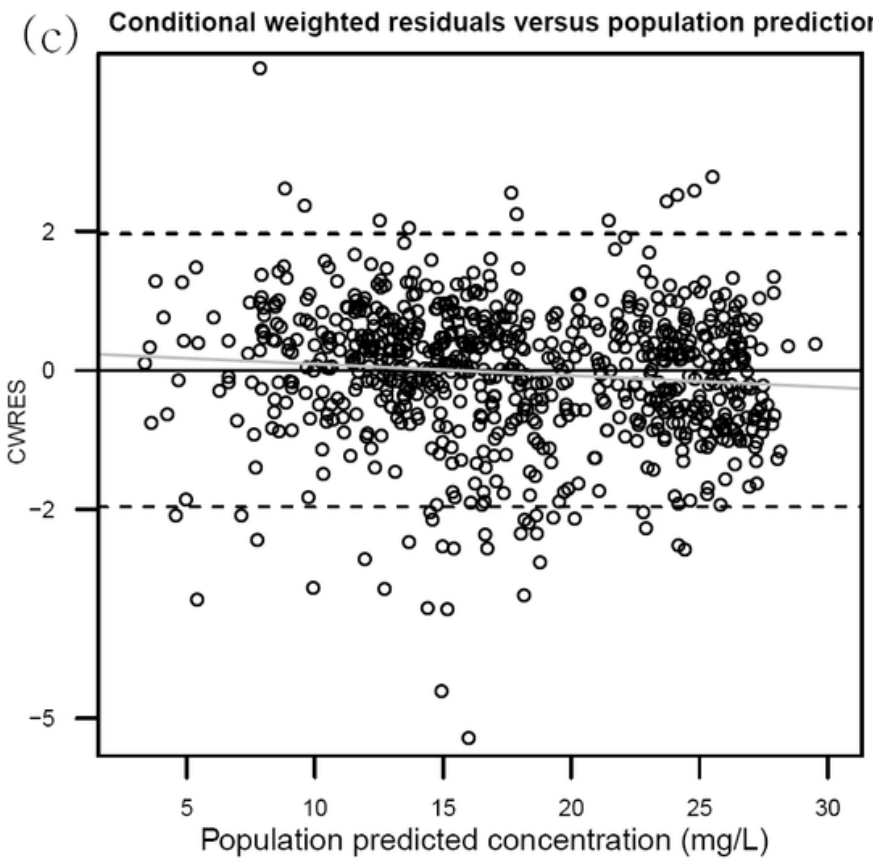

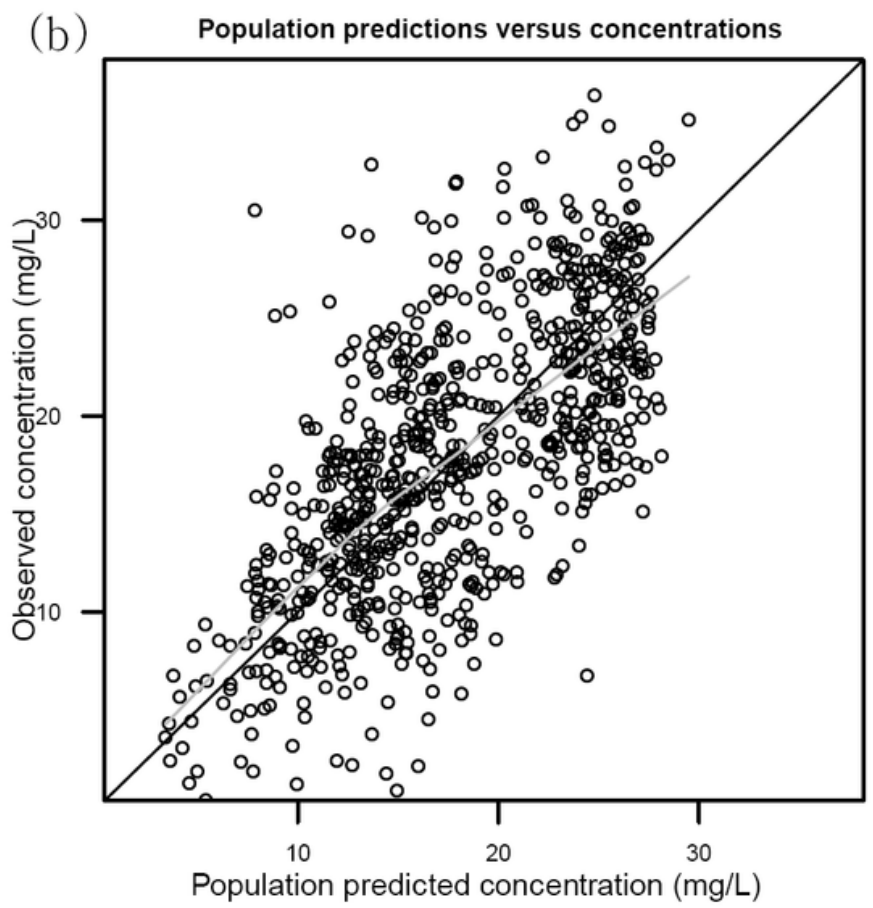

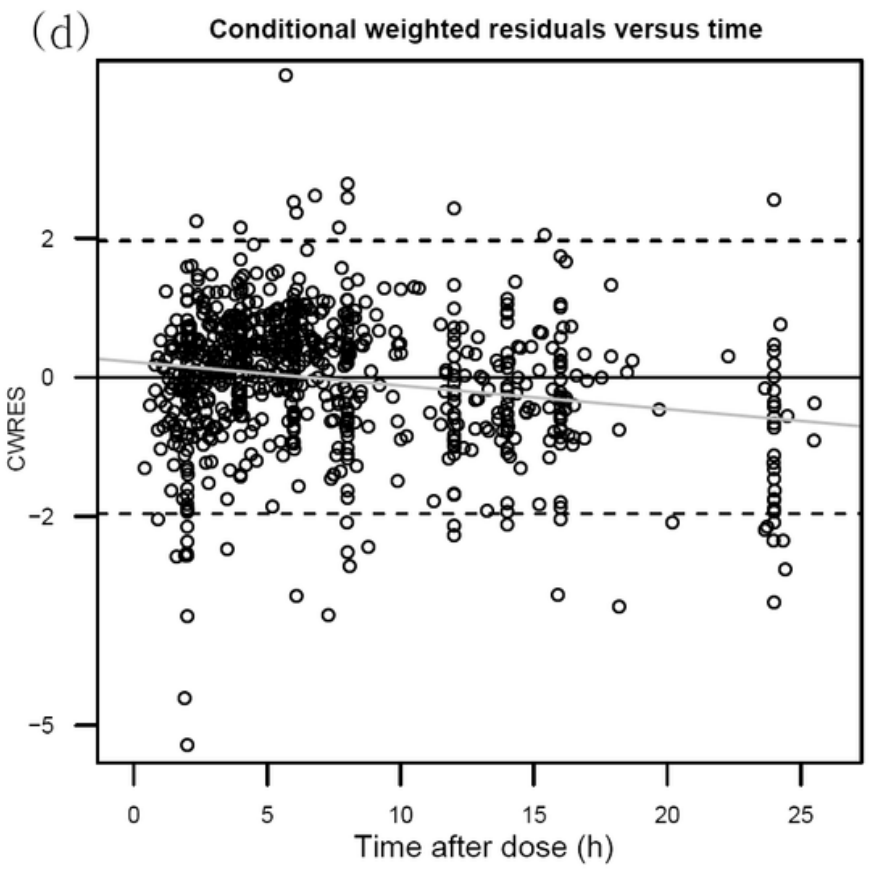

\section{Figure 1}

Basic goodness-of-fit plots of final model. (a) Observed values versus individual predicted values; (b) observed values versus population predicted values; (c) conditional weighted residuals (CWRES) versus population predicted values; and (d) CWRES versus time. Black and grey solid lines indicate the line of identity and Loess (locally weighted smoothing), respectively. 
(a)

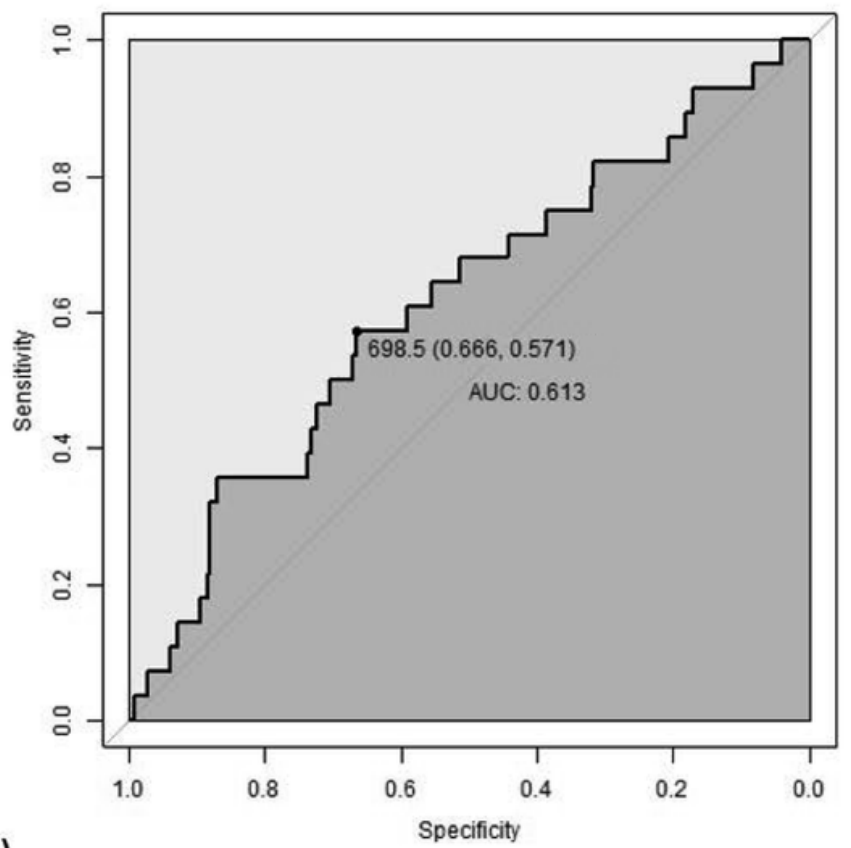

(c)

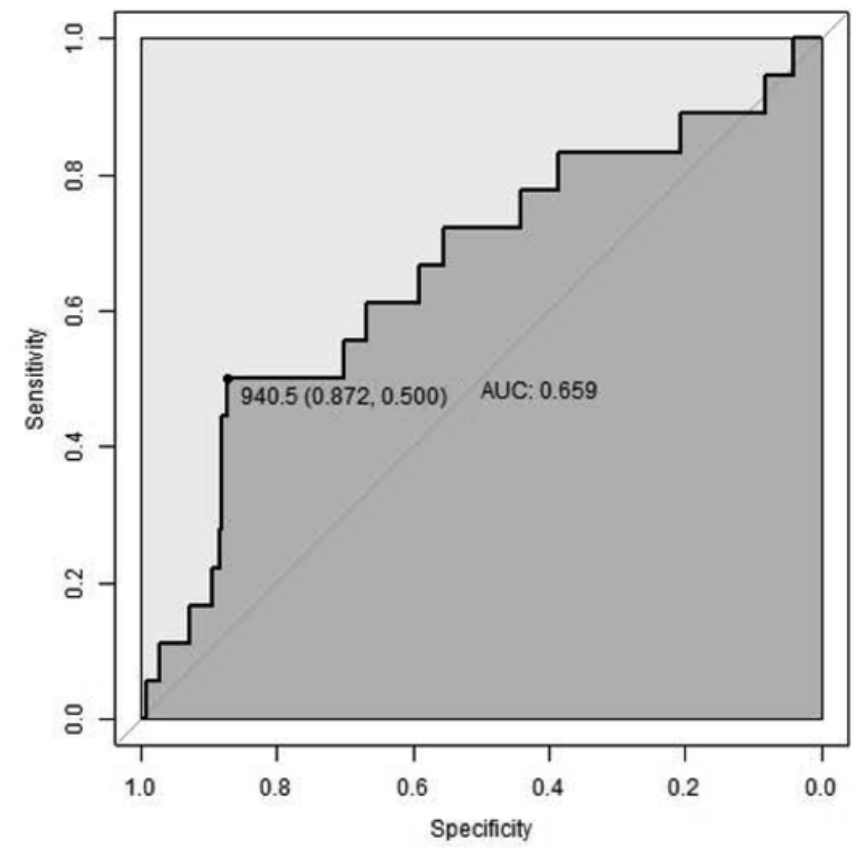

(b)

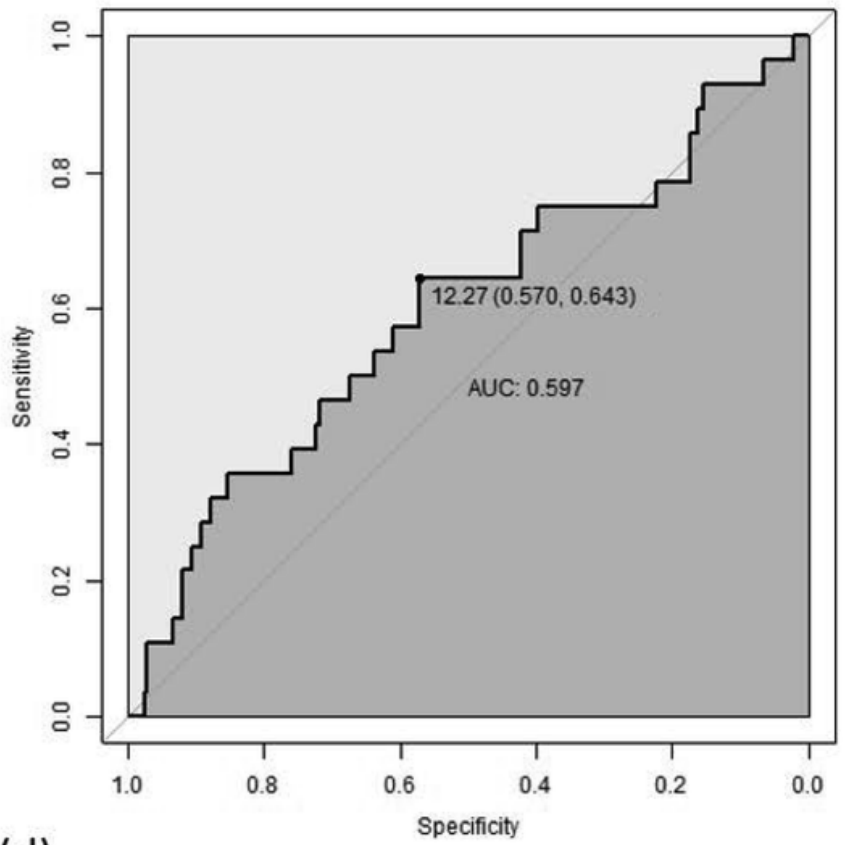

(d)

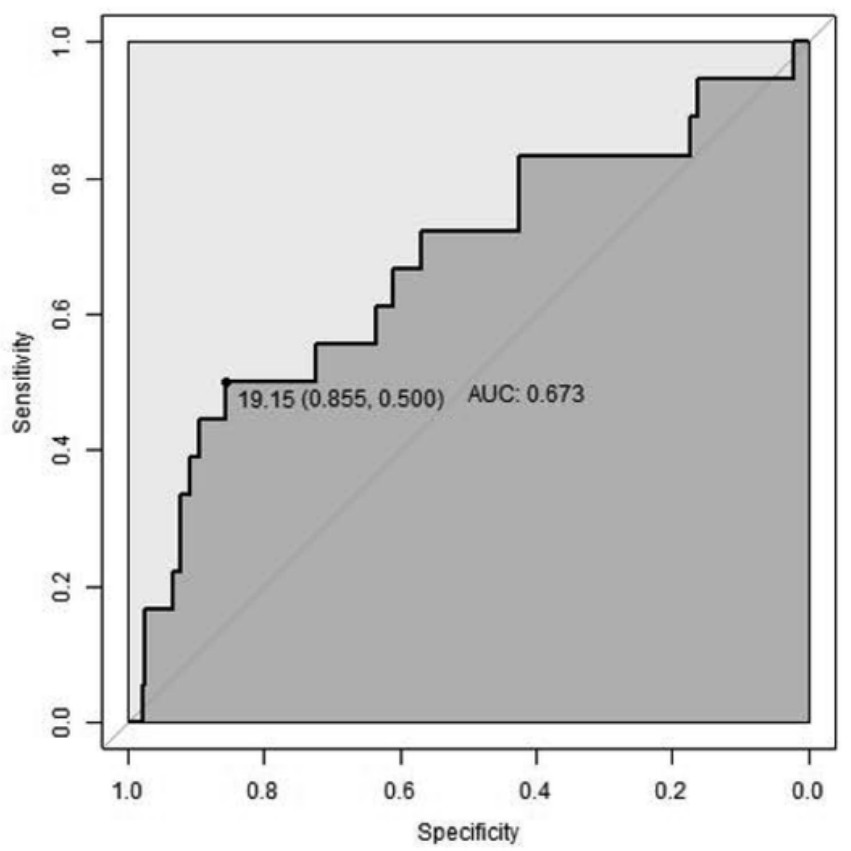

Figure 2

ROC curve for MHD levels in predicting adverse events of oxcarbazepine. (a) MHD AUC in predicting doserelated adverse events; (b) MHD trough levels in predicting dose-related adverse events; (c) MHD AUC in predicting dizziness occurrence; (d) MHD trough levels in predicting dizziness occurrence; ROC, receiveroperator characteristics; AUC area-under the curve. The data are presented as MHD levels (specificity, sensitivity) 


\section{Supplementary Files}

This is a list of supplementary files associated with this preprint. Click to download.

- Supplementaryfile.docx

- Supplementaryfigure1.pdf 